\title{
Hippocampal Shape Analysis Using Medial Surfaces
}

\author{
Sylvain Bouix ${ }^{1}$, Jens C. Pruessner ${ }^{2}$, Donald L. Collins ${ }^{2}$, and Kaleem Siddiqi ${ }^{1}$ \\ ${ }^{1}$ Centre for Intelligent Machines \& School of Computer Science \\ 2 McConnell Brain Imaging Centre, Montreal Neurological Institute \\ McGill University, Montreal, Canada
}

\begin{abstract}
Within the medial temporal lobe, significant attention has been paid to the analysis of the hippocampus (HC) in MR images because of its intimate connection to memory, emotion and learning. Volume changes in the HC have been recorded in conjunction with Alzheimer's disease, post-traumatic stress disorder and depression. Recent studies have also found a significant reduction in $\mathrm{HC}$ volume that is related to gender; it is found in men but not women. In this paper we demonstrate a shape analysis of the $\mathrm{HC}$ and employ it to investigate gender differences in normal subjects. For each subject we extract the dominant medial sheet of the HC, find the plane defined by its two principal eigen vectors and then express the medial surface radius as a height function over this plane. This allows us to statistically quantify the relationship between several independent variables and local object width.
\end{abstract}

\section{Introduction}

In studies employing hippocampus (HC) and amygdala (AG) segmentation based on magnetic resonance (MR) imaging, varying degrees of volume loss in conjunction with Alzheimer's disease, depression and post-traumatic stress disorder have been observed. The precise nature of this loss and its connection to variables such as age and gender remains somewhat controversial, pointing to the need for better quantitative models of $3 \mathrm{D}$ shape to be used in this context. With respect to structures such as the $\mathrm{HC}$, a good candidate is the medial surface. The most prominent medial surface sheet can be used to register individual data sets, following which more precise comparisons can be made [13].

Medial models have been successfully used in medical image analysis in a number of contexts, e.g., see [7/4 for some recent applications. Applying these methods in $3 \mathrm{D}$ presents a challenge because only a small class of computationally reliable algorithms exist. One such class relies on pruning strategies for $3 \mathrm{D}$ Voronoi diagrams [1,6]. This has recently been used in conjunction with a boundary description to provide a characteristic model for 3D shape analysis [13]. An important idea is the use of a medial primitive 8 to describe the most prominent medial sheet and provide an intrinsic frame of reference by which different data sets can be registered. 
In this paper we adopt a similar strategy, but use a novel algorithm we have developed for computing medial surfaces. Our main goal is to apply this methodology to a data set for which volume loss in relation to gender and age has been previously investigated [9]. The main idea is to extract the dominant medial sheet of the $\mathrm{HC}$ for each subject, find the plane defined by its two principal eigen vectors and then express the medial surface radius as a height function over this plane. This in turn allows us to statistically examine the relationship of independent variables with local object width.

\section{Divergence Based Medial Surfaces}

In this section we review the algorithm for computing medial surfaces 112].

\subsection{The Hamilton Jacobi Formulation}

Consider the grassfire flow

$$
\frac{\partial S}{\partial t}=\mathcal{N}
$$

acting on a closed 3D surface $S$, such that each point on its boundary is moving with unit speed in the direction of the inward normal $\mathcal{N}$. In physics, such equations are typically solved by looking at the evolution of the phase space of an equivalent Hamiltonian system. Let $D$ be the Euclidean distance function to the initial surface $\mathcal{S}_{0}$. The magnitude of its gradient, $\|\nabla D\|$, is identical to 1 in its smooth regime. The associated Hamiltonian system is given by:

$$
\dot{\mathbf{p}}=(0,0,0), \quad \dot{\mathbf{q}}=-\left(D_{x}, D_{y}, D_{z}\right) \text {. }
$$

Hamiltonian systems are conservative and hence the vector field $\dot{\mathbf{q}}$ is divergence free. However, this property does not hold at singularities of $\dot{\mathbf{q}}$, which coincide with medial surface points. We use the divergence theorem to measure the net outward flux of $\dot{\mathbf{q}}$ through a surface

$$
\int_{v} \operatorname{div}(\dot{\mathbf{q}}) \mathrm{dv} \equiv \int_{\mathrm{S}}<\dot{\mathbf{q}}, \mathcal{N}>\mathrm{ds} .
$$

In the limit as the volume enclosed by the surface shrinks to zero we obtain a numerical approximation to the divergence which is used to guide a thinning process in a cubic lattice, while taking care to preserve the object's topology.

\subsection{Preserving Homotopy}

A point is a simple point if its removal does not change the topology of the object. Hence in 3D, its removal must not disconnect the object, create a hole, or create a cavity. Malandain et al. have introduced a topological classification of a point $x$ in a cubic lattice by computing two numbers [5]: i) $C^{*}$ : the number of 26-connected components 26-adjacent to $x$ in $O \cap N_{26}^{*}$, and ii) $\bar{C}$ : the number 
of 6-connected components 6-adjacent to $x$ in $\bar{O} \cap N_{18}$. Further, they have shown that if $C^{*}=1$ and $\bar{C}=1$, the point is simple, and hence removable.

The basic strategy now is to guide the thinning of the object by the total outward flux measure computed over a very small neighborhood. Points with the most negative outward flux are the strongest medial surface points. The process is stopped when all surviving points are not simple, or have a total outward flux below some chosen (negative) value, or both. Unfortunately the result is not guaranteed to be a thin set, i.e., one without an interior.

This last constraint can be satisfied by defining an appropriate notion of an endpoint in a cubic lattice. In $\mathcal{R}^{3}$, if there exists a plane that passes through a point $p$ such that the intersection of the plane with the object includes an open curve which ends at $p$, then $p$ is an end point of a 3D curve, or is on the rim or corner of a 3D surface. This criterion can be discretized easily to 26 -connected digital objects by examining 9 digital planes in the 26-neighborhood of $p$ 10]. The thinning process proceeds as before, but the threshold criterion for removal is applied only to endpoints.

\subsection{Segmenting the Medial Surface}

The medial surface can now be segmented using the classification of [5]. Specifically, the numbers $C^{*}$ and $\bar{C}$ can be used to classify curve points, surface points, border points and junction points. However, junction points can be misclassified as surface points when certain special configurations of voxels occur. These cases have to be dealt with using a new definition for simple surfaces [5].

Let $x$ be a surface point $\left(\bar{C}=2\right.$ and $\left.C^{*}=1\right)$. Let $B_{x}$ and $C_{x}$ be the two connected components of $\bar{O} \cap N_{18} 6$-adjacent to $x$. Two surface points $x$ and $y$ are in an equivalence relation if there exists a 26-path $x_{0}, x_{1}, \ldots, x_{i}, \ldots, x_{n}$ with $x_{0}=x$ and $x_{n}=y$ such that for $i \in[0, \ldots, n-1],\left(B_{x_{i}} \cap B_{x_{i+1}} \neq \emptyset\right.$ and $\left.C_{x_{i}} \cap C_{x_{i+1}} \neq \emptyset\right)$ or $\left(B_{x_{i}} \cap C_{x_{i+1}} \neq \emptyset\right.$ and $\left.C_{x_{i}} \cap B_{x_{i+1}} \neq \emptyset\right)$. A simple surface is defined as any equivalence class of this equivalence relation.

We use this definition in our framework to find all the different simple surfaces comprising the medial surface of an object. The first unmarked surface point on the medial surface is found and used as a "source" to build its corresponding simple surface using a depth first search strategy. The next simple surface is built from the next unmarked point and so on, until all medial surface points are marked.

\section{Hippocampal Data}

In a previously published analysis [9, the left and right $\mathrm{HC}$ and $\mathrm{AG}$ were manually segmented from T1-weighted MR images (three dimensional spoiled gradient echo acquisition with sagittal volume excitation; repetition time 18, echo time 10, flip angle 30 degrees) from 80 normal healthy subjects. These subjects included 39 healthy men and 41 healthy women in the age range of 18 to 42 years (mean age $25.4+/-5.6$ years). The MRI data for each subject was first corrected for 
image intensity non-uniformity [12] and linearly registered to a standard brainbased coordinate system known as stereotaxic space [3]. It was then segmented manually by $3 \mathrm{D}$ voxel painting. By resampling with a tri-cubic kernel onto a $1 \mathrm{~mm}^{3}$ voxel grid in stereotaxic space, each brain volume was normalized for size, orientation and position. A typical segmentation of an individual hippocampus (HC) labelling is shown in Fig. 1

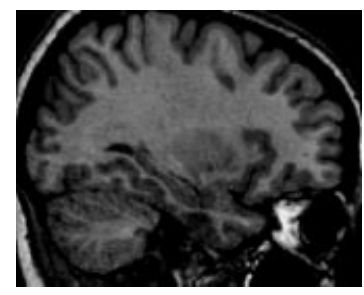

(A)

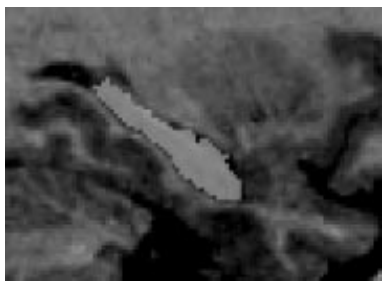

(B)

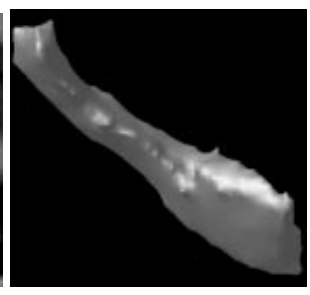

$(\mathrm{C})$

Fig. 1. Hippocampal data from one subject. (A) Sagittal T1-weighted MRI image, (B) zoomed sub-image centred on hippocampus with hippocampal label, (C) $3 \mathrm{D}$ view of the manually segmented hippocampus.

Quantitative analysis of the original label volumes derived from the MR images revealed an overall hemispheric difference with the right $\mathrm{HC}$ being bigger than the left (4300 vs. $4072 \mathrm{~mm}^{3}, p<.001$ ). Furthermore, there was a correlation with age in the group of men for both left and right hippocampus (Spearman's $r=-.44, p<.005)$ that was not apparent in the group of women $(r=.01, p>$ $.20)$. In order to determine the location of these differences, a regression analysis was applied to each voxel, with image intensity as the dependent variable [9]. Since nearby voxels were expected to be correlated, the technique of Worsley et al. 14 was used to determine the critical $t$-threshold to identify statistically significant regressions. The analysis revealed that the differences between men and women seemed to occur mostly in the head and tail of the HC. However, it was not possible to determine whether the observed differences were due only to a volume reduction or were associated with the overall shape of the $\mathrm{HC}$ as well. The medial surface algorithm presented here extends the possibilities for analysis, allowing the investigation of shape differences in the $\mathrm{HC}$ between men and women. We chose to first examine the radius function of the medial surface of the $\mathrm{HC}$ at different locations in the human brain. It was thus necessary to register all the $\mathrm{HC}$ data sets using a local common coordinate frame, such that comparisons between subjects and groups could be made.

Our experiments showed that across the full set of $\mathrm{HC}$ data, the medial surface consisted of one large prominent sheet with only a small number of additional sheets. Furthermore, with only one exception, the volume reconstructed by the prominent sheet was less than $1 \%$ different than that reconstructed by the entire medial surface. We also found that the prominent sheet was almost 
planar: typical eigenvalue magnitudes for the principal component directions were $\lambda_{1}=100, \lambda_{2}=20, \lambda_{3}=1$. This justified a strategy of projecting the main medial surface sheet onto the plane defined by its two principal axes and then storing desired information about the object (the radius function of the medial surface) as a $\mathcal{R}^{2} \rightarrow \mathcal{R}$ height function. This process is illustrated in Fig. 2, In summary, the steps involved are: 1. Compute the medial surface of the HC, 2 . Extract the largest simple surface as a 2D manifold in 3D, 3. Compute the two principal component axes of this manifold, 4. Project all points on the manifold onto the $2 \mathrm{D}$ plane with axes given by the principal component axes and origin given by the projection of the manifold's centroid, and 5. For each projected point display the medial surface radius as a height function mapping $\mathcal{R}^{2} \rightarrow \mathcal{R}$.

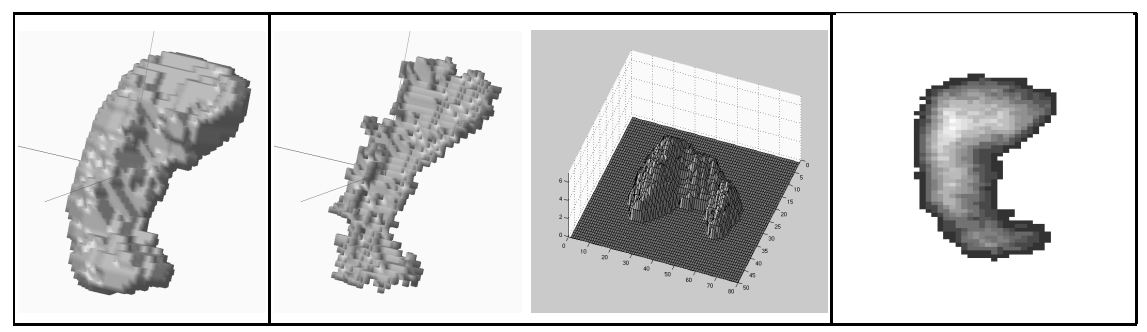

Fig. 2. Left to Right: A hippocampus, the most prominent sheet of its medial surface, the medial surface radius function as a surface plot, and as an intensity map.

\section{Analysis}

The computation of the medial surfaces from the original $\mathrm{HC}$ labels resulted in two dimensional maps with each point on the map referring to the medial surface radius as a height function (Fig. 3-A). These individual $2 \mathrm{D}$ maps could then be averaged to compute the mean medial surface map with radius information for both men and women (e.g., Fig. 3 B and C). Both the single and the average maps could be employed for further statistical analysis. For example, one can see subtle differences in the left and right average maps in Fig. 3 B and $\mathrm{C}$. The voxelbased regression technique could then be applied to compute an age corrected t-statistic image to localize statistically significant differences (Fig. [3-D).

Employing the medial surfaces in a linear regression model with gender as the independent variable, age as the covariate and the individual points on the 2D map as dependent variables, the slope of the effect of gender on the medial surface of the HC could be computed. The results of this analysis (Fig. 4) reveal differences in the medial surfaces between left and right hemispheres as well as between men and women. First, it appears that there is a stronger effect of 


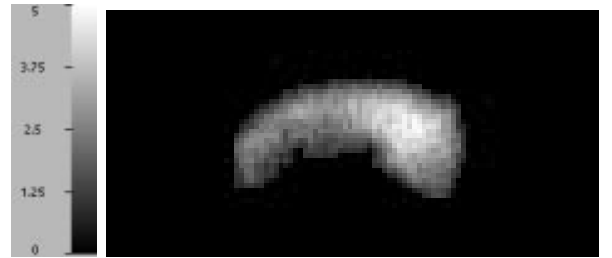

(A)

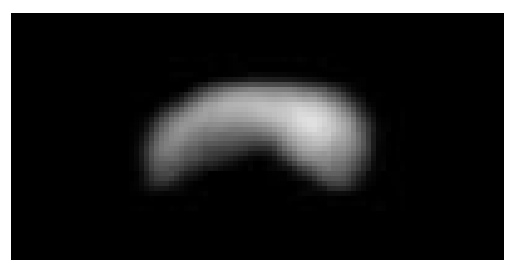

(C)

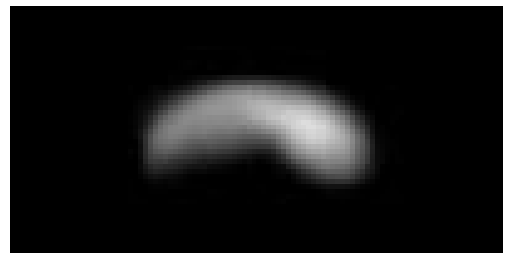

(B)

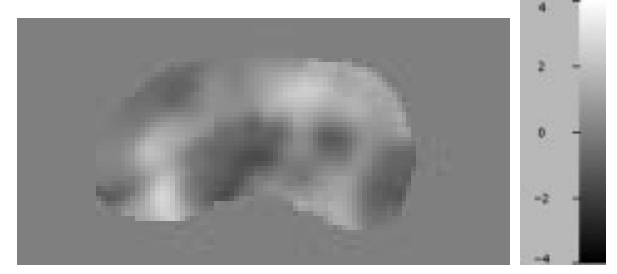

(D)

Fig. 3. Horizontal section of medial surface radius function, anterior at the right, posterior at the left and medial at the top of image. (A) Radius function projected onto a 2D sheet for one subject. The grey bar (left side) indicates a radius of 0 to $5 \mathrm{~mm}$. (B) Average radius function for left female hippocampus. This image is created by computing the average, on a pixel-by-pixel basis, of the radius function of the left $\mathrm{HC}$ for the 41 women in the study. One can see that on average the head of the $\mathrm{HC}$ (right side, image B) is approximately $10 \mathrm{~mm}$ thick from top to bottom and that the tail (left side) gradually decreases in thickness. (C) Average radius for right female hippocampus. There appears to be a difference between the left and right sides. In order to localize statistically significant differences, a pairwise age corrected t-statistic image was computed for right-left (D). High intensity areas indicate regions where the right side is larger than the left.

gender and age on the right hemisphere than on the left; the resulting t-values are higher in the right hemisphere. Adjacent regions of positive and negative tvalues indicate possible local translation. Evidence for this effect at the head and tail of the right hemisphere $\mathrm{HC}$ indicate a rotation of female $\mathrm{HC}$ with respect to the male $\mathrm{HC}$, with womens' $\mathrm{HC}$ head moving laterally and the tail moving medially. However, the difference in magnitude between the two regions suggests differences in the size of the medial anterior section of the $\mathrm{HC}$ between males and females. We note that the recent study in [13] reports that the right $\mathrm{HC}$ is on average thicker than the left, although the connection to gender was not examined.

Second, Fig. 5 shows the individual age regression maps for the 4 groups. The intensity of the image is directly proportional to the magnitude (and sign) of the regression of the medial surface radius function with age and thus reveals information about the effect of age on the location of shape differences. The 


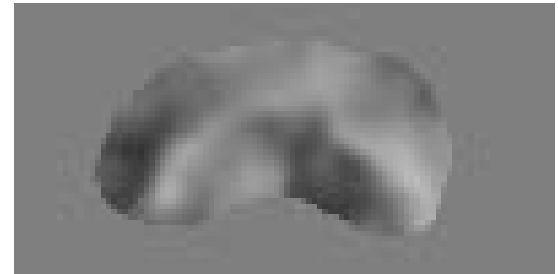

(A)

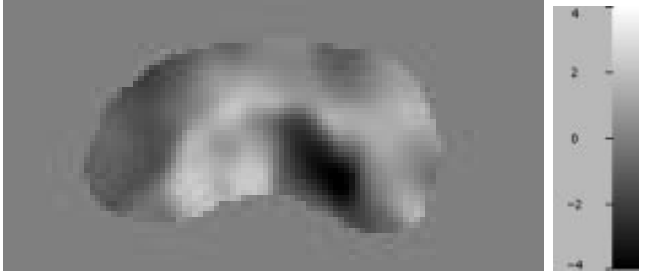

(B)

Fig. 4. Regression of gender, correcting for age, left (A) and right (B) hemispheres. The grey bar indicates a range of t-values from -4.0 to 4.0 .

most apparent result is that the regressions are stronger in men than in women, and stronger on the right side in both sexes. We can see positive correlations in the medial anterior region of $(\mathrm{A})$, suggesting a thickening of the alveus with age in females. In males, there is a significant decrease in the anterior and posterior regions, corresponding to the uncal recess and the inferior horn of the lateral ventricles. These results confirm those found in our previous study 9 .

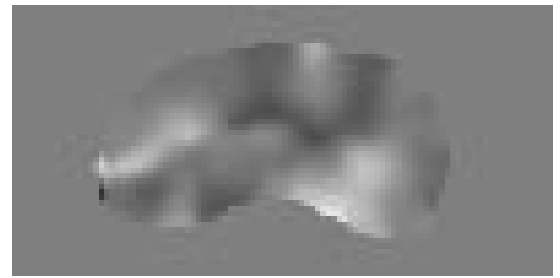

(A)

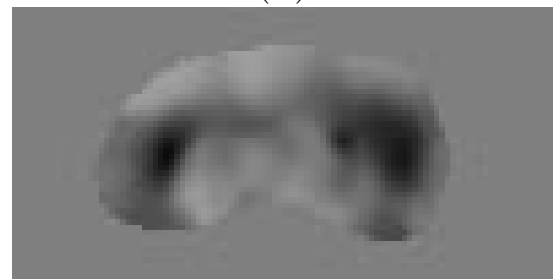

(C)

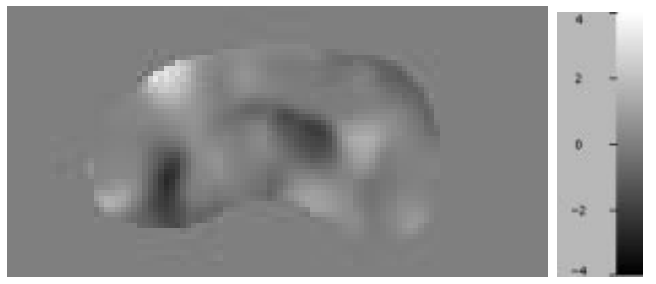

(B)

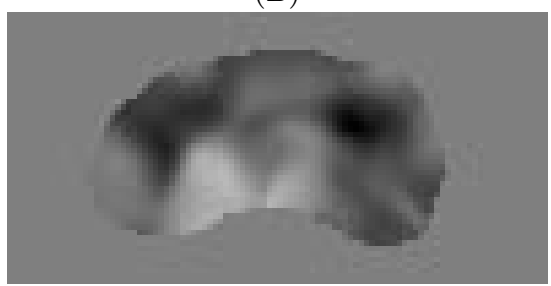

(D)

Fig. 5. Regression of age for women left (A) and right (B) hemispheres; and men left $(\mathrm{C})$ and right (D). The grey bar indicates t-values from -4.0 to 4.0 . 


\section{Conclusions}

Our experiments indicate that association with $\mathrm{HC}$ shape is stronger in men. This confirms our previous results which showed a stronger (negative) correlation of volume with age in men than in women. Future studies need to address the association between shape and volume more precisely, possibly by adding volume information as a covariate in the voxel-based regression analysis. The use of a medial surface model provides the advantage that other aspects of $\mathrm{HC}$ shape (such as boundary curvature) can also be incorporated as a height function, allowing for further quantitative analysis.

\section{References}

1. D. Attali, G. S. di Baja, and E. Thiel. Skeleton simplification through nonsignificant branch removal. Image Processing and Communications, pages 63-72, 1997.

2. S. Bouix and K. Siddiqi. Divergence-based medial surfaces. In ECCV'2000, pages 603-618, Dublin, Ireland, June 2000.

3. D. L. Collins, P. Neelin, T. M. Peters, and A. C. Evans. Automatic 3d intersubject registration of $\mathrm{mr}$ volumetric data in standardized talairach space. Journal of Computer Assisted Tomography, 18:192-205, 1994.

4. P. Golland, W. E. L. Grimson, and R. Kikinis. Statistical shape analysis using fixed topology skeletons: Corpus callosum study. In IPMI'1999, 1999.

5. G. Malandain, G. Bertrand, and N. Ayache. Topological segmentation of discrete surfaces. International Journal of Computer Vision, 10(2):183-197, 1993.

6. M. Näf, O. Kübler, R. Kikinis, M. E. Shenton, and G. Székely. Characterization and recognition of $3 \mathrm{~d}$ organ shape in medical image analysis using skeletonization. In IEEE Workshop on Mathematical Methods in Biomedical Image Analysis, 1996.

7. S. M. Pizer, D. S. Fritsch, P. Yuskhevich, V. Johnson, and E. Chaney. Segmentation, registration and measurement of shape variation via image object shape. IEEE Transactions on Medical Imaging, 18:851-865, 1999.

8. S. M. Pizer, A. Thall, and D. T. Chen. M-reps: A new object representation for graphics. ACM Transactions on Graphics (submitted), 1999.

9. J. C. Pruessner, D. L. Collins, M. Pruessner, and A. C. Evans. Age and gender predict volume decline in the anterior and posterior hippocampus in early adulthood. The Journal of Neuroscience, To appear, 2001.

10. C. Pudney. Distance-ordered homotopic thinning: A skeletonization algorithm for 3d digital images. Computer Vision and Image Understanding, 72(3):404-413, 1998.

11. K. Siddiqi, S. Bouix, A. Tannenbaum, and S. W. Zucker. The hamilton-jacobi skeleton. In ICCV'99, pages 828-834, Kerkyra, Greece, September 1999.

12. J. G. Sled, A. P. Zijdenbos, and A. C. Evans. A non-parametric method for automatic correction of intensity non-uniformity in mri data. IEEE Transactions On Medical Imaging, 17(1):87-97, 1998.

13. M. Styner and G. Gerig. Medial models incorporating object variability for $3 \mathrm{~d}$ shape analysis. In IPMI'2001, pages 502-516, 2001.

14. K. J. Worsley, J. B. Poline, and A. C. Evans. Characterizing the response of pet and fmri data using multivariate linear models. NeuroImage, 6:305-319, 1998. 